\title{
Movement of the wagon on the marshalling hump under the impact of air environment and tailwind
}

\author{
Khabibulla Turanov ${ }^{1, *}$, Andrej Gordienko ${ }^{2}$, Shukrat Saidivaliev ${ }^{3}$, Shukrat Djabborov ${ }^{3}$ \\ ${ }^{1}$ Tashkent state technical University named Islam Karimov, 2, University Str., 100174, Tashkent, \\ Uzbekistan \\ ${ }^{2}$ Ural State University of Railway Transport, 66, Kolmogorova Str., 620034, Yekaterinburg, Russia \\ ${ }^{3}$ Tashkent Railway Engineering Institute, 1, Temirylchilar Str., 100167, Tashkent, Uzbekistan
}

\begin{abstract}
The effect of the tailwind on the wagon when it is rolling down from the hump. Study of the movement of the wagon on the profile of the marshalling hump. To provide an incorrect formula for determining the relative velocity and air particles, taken as the absolute velocity of the particles. The article applies the theorem on the addition of velocities in complex motion in vector form. Mathematical substantiated errors in the determination of the specific resistance to movement of the wagon from the air medium and wind, derived from the empirical relationships given the aerodynamic performance of the wagons. The results of the research can be used in the processing of the normative-technical document on the design of hump devices on railways and making adjustments to the dynamics of rolling the wagon in textbooks for universities of railway transport. The results of this study prove the need to revise the practical significance of the formula for finding the specific resistance to the movement of the car from the air and wind in the in the normativetechnical document "Rules and regulations for the design of sorting devices on the railways track gauge $1520 \mathrm{~mm} "$.
\end{abstract}

\section{Introduction}

The problems of designing marshalling hump are studied in [1 - 24]. This paper is a continuation of a series of publications on the dynamics of sliding of the wagon on the drain side hump when exposed to a force projection tailwind [17 - 19]. Particularly, make a reservation that the eleventh counterexample presented in [17], for some unknown reason, was not discussed by the authors [18, 19] in favor of the correctness of the theoretical propositions of the dynamics of rolling the wagon down the slope of the hump yard. However, in [18] noted that, "according to the authors of the article [17], the formula for determining the resistivity from the air environment $\mathrm{w}_{\mathrm{ae}}$ is "incorrect", but there is no the response about the error [12]. Therefore, it is groundless statement" (see also p. 24 in [18]). This paper will discuss in more detail the possible reason for the lack of mathematically justified and confirmed by results of calculations of the counter-arguments in the eleventh

* Corresponding author: khturanov@yandex.ru 
counterexample in [17]. To do this, the results of the research in [17] are given, by definition, the resulting speed $v_{\text {res }}$, is equal to the sum of the wind velocity $\mathrm{v}_{\mathrm{w}}$ and the vector $\mathrm{v}_{\mathrm{cg}}$, equal in magnitude to the speed of the car $\mathrm{v}_{\mathrm{car}}$, but opposite in direction, the mathematics of which [without explanation, the direction of the vector $v_{c r}$, "opposite in direction of the velocity of the car $v_{\text {car }}$ "] represented in the form (see formula (13) in the eleventh counterexample [17]):

$$
v_{r}^{2}=v_{c g}^{2}+v_{c}^{2} \pm 2 v_{c g} v_{c} \cos \beta
$$

where $\left|v_{c g}\right|=v_{c a r}-$ is the average speed of the wagon found in its input $v_{i}$ and the output $v_{i}+$ 1 from the measuring area hump;

$\beta$ - the angle between the wind direction and the axis of the path segment ( $\mathrm{x}$-axis) (always less than $90^{\circ}$ ).

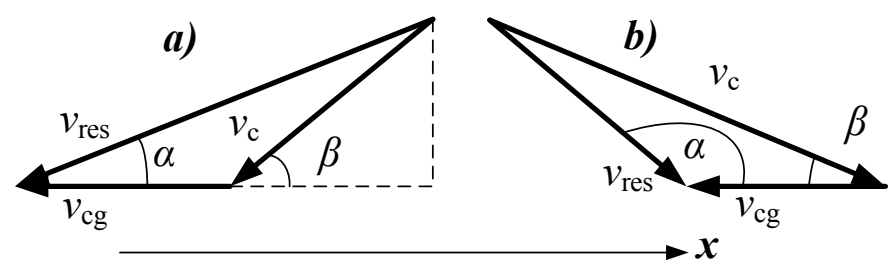

Fig. 1. Computing scheme of the resulting vector and angle:

a) - with a headwind; b) - with the tailwind; $\mathrm{x}$-axis - direction movement of the wagon

Note that in (1) the «plus» sign corresponds to the oncoming wind, and «minus»direction of tailwind.

In the eleventh counterexample [17] the angle $\alpha$ between the resultant wind velocity $v_{\text {res }}$ and the axis of the path $\mathrm{x}$ (see Fig. 1) was calculated according to the theorem of sines, in the form:

$$
\alpha=\arcsin \left(\frac{v_{c}}{v_{\text {res }}} \sin \beta\right) .
$$

In the next formula (1) to determine the resultant wind speed of $\mathrm{v}_{\text {res }}$ was used to obtain the empirical formula of the specific air resistance of the wagon $\mathrm{w}_{\mathrm{ar}}$ (see formula (12) in ninth counterexample [17]) in the form $\mathrm{kgf} / \mathrm{t}$ :

$$
w_{a r}=\frac{17.8 C_{x} s}{\left(273+t^{\circ}\right) q} v_{r e s}^{2}
$$

where 17.8 - constant value found on the basis of experimental data taking into account the air density $\rho$ (in $\mathrm{kg} / \mathrm{m}^{3}$ ) relative to the atmospheric pressure (e.g., in the range of $700 \ldots$ $780 \mathrm{~mm}$ of mercury column) and the ambient temperature $\mathrm{t}$;

$\mathrm{C}_{\mathrm{x}}$ - the coefficient of air resistance (usually depending on the angle $\alpha$ (e.g., $\alpha$ from 0 to $90^{\circ}$ ) varies from $1.2 \ldots 1.9$ (dimensionless quantity);

$\mathrm{s}$ - the cross-sectional area (maximum cross-section) of the wagon (e.g. for four-axle platform is 4.1 , and for four-axle high-sided wagon $-8.5, \mathrm{~m}^{2}$;

$\mathrm{q}$ - the weight of the wagon, $\mathrm{t}$ (not the vehicle); 
$\mathrm{t}^{\circ}-$ ambient temperature, ${ }^{\circ} \mathrm{C}$;

$273+\mathrm{t}^{\circ}=\mathrm{T}$ is the absolute ambient temperature, $\mathrm{K}$ (Kelvin scale);

$\mathrm{v}_{\mathrm{p}}$ - the resultant speed determined by the formula (2), $\mathrm{m} / \mathrm{s}$.

Note that because the formula (3) is empirical, it is virtually not possible to verify the correctness of its dimension in $\mathrm{kgf} / \mathrm{t}$.

Especially stipulate that in Fig. 1, the vector $\mathrm{v}_{\mathrm{cr}}$, without explanation, directed opposite to the direction of the velocity of the wagon $\mathrm{v}_{\mathrm{car}}$ that corresponds to the theorem of addition of velocities with the complex movement of theoretical mechanics [24]. In [18] it is stated that "before you decide to criticize made earlier, it is necessary to examine theoretical approaches and practical engineering methods at least the leading schools in the design of hump yards" (see the first paragraph of middle column on p. 24 in [18]). In [17] it is noted that the formula (3) is empirical. However, in this case, the velocity $v_{p}$ is an unknown quantity. All this is one of the main reasons that the formula for determining the resistivity from the air environment $\mathrm{w}_{\mathrm{ae}}$ (3) in [17] is marked as "incorrect ". For a detailed proof of "gross errors" formula (1) and (2), and, consequently, (3), containing the resultant speed $\mathrm{V}_{\text {res}}$, will conduct the study, based on the correct application of the theorem on composition of velocities in complex movement theoretical mechanics.

\section{Goal of research}

- To prove the incorrectness of the formula for determining the relative speed of air particles, which is taken as the absolute velocity of the particles (i.e. the wind speed relative to the ground), which contradicts the principle of relative motion of a material point in classical mechanics;

- To justify gross errors in the determination of the specific resistance to movement of the wagon from the air environment and tailwind derived from the empirical correspondence given the aerodynamic properties (streamlined) wagons, and given in the regulatory-technical documents.

- To give examples of calculation for evaluating the correctness and/or incorrectness of performed analytical studies on the construction of mathematical models of the motion of the wagon on exposure to tailwind and to compare the values obtained with the use of the regulatory-technical document.

\section{Research methods}

Research methods rely on the use of the theorem of velocity addition in a complex motion in vector form [24].

\section{The main results of the study}

It is known [7, $13-15,20,21]$, on the hump the automatic shunting is carried out from the top with initial speed $v_{i v}$. Therefore, with the top of the hill (In G) can be linked stationary coordinate system $\mathrm{O}_{1} \mathrm{x}_{1} \mathrm{y}_{1} \mathrm{z}_{1}$ (Fig. 2). In this case we assume that the point $\mathrm{M}$ (for example, movement of air particles and wind) over some time t for some moving mobile reference system Oxyz, which, in turn, moves relative to the main (fixed) coordinate system $\mathrm{O}_{1} \mathrm{x}_{1} \mathrm{y}_{1} \mathrm{z}_{1}$ (Fig. 2). Then, as is well known [24], the trajectory of the point M, considered in relation to the system Oxyz, is the path relative (or local) motion, and in relation to $\mathrm{O}_{1} \mathrm{x}_{1} \mathrm{y}_{1} \mathrm{Z}_{1}-$ absolute (or full) motion. Motion of the movable system Oxyz in relation to the fixed $\mathrm{O}_{1} \mathrm{X}_{1} \mathrm{y}_{1} \mathrm{Z}_{1}$ is for a moving point $\mathrm{M}$ portable movement. The speed of a moving point $\mathrm{M}$ in 
relation to the system Oxyz called relative velocity $\bar{v}_{r}$, and relative to the system $\mathrm{O}_{1} \mathrm{x}_{1} \mathrm{y}_{1} \mathrm{z}_{1}-$ absolute speed $\bar{v}_{a}$. The speed of that invariably is associated with a moving frame of reference Oxyz in terms of space, in which the time $\mathrm{t}$ is a moving point $\mathrm{M}$, called the transportation velocity $\bar{v}_{e}$ [24].

On this basis, in relation to the movement of the wagon down the profile of the hump it may be noted that the carriage moving at a speed of $\bar{v}_{c a r}=\bar{v}$, can test the effect of the air environment and tailwind (as in Fig. 2). Therefore, from any point of the wagon (for example, point $\mathrm{O}$ ) movable coordinate system Oxyz can be linked (Fig. 2 applicate $\mathrm{z}$ perpendicular to the plane of the drawing, is not shown).

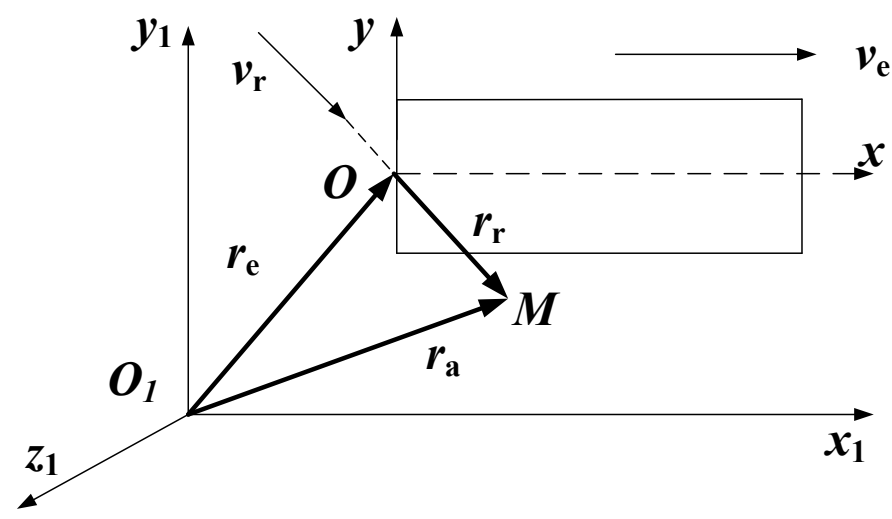

Fig. 2. The direction of the vectors-radius of the wagon and wind (air particles).

Note that Fig. 2 is a schematic, because it is not possible to show fully functional movement of the point $M$ relatively to moving reference system Oxyz, and the latter relative to a fixed reference system $\mathrm{O}_{1} \mathrm{x}_{1} \mathrm{y}_{1} \mathrm{z}_{1}$. For better understanding of the complex motion of the point $\mathrm{M}$ in Fig. 2 is indicated: $\bar{r}_{e}$ - the vector-radius of the portable motion (motion of the wagon); $\bar{r}_{r}$ - the vector-radius of the relative motion of air particles $\mathrm{M}$ (see pp. $59-60$ in [24]); $\bar{r}_{a}$ - vector-radius of the absolute motion of air particles $M$ relative to the earth (absolute motion of air particles M) (Fig. 2); $\bar{v}_{e}-$ the direction of the velocity of movement of the wagon; $\bar{v}_{r}-$ the direction of the relative speed of air particles $\mathrm{M}$.

When the complex movement of the vector-radius of the absolute motion of the particles of air $\mathrm{M}$ is equal to the geometric sum of the vector-radius of the transportation and relative motion:

$$
\bar{r}_{a}=\bar{r}_{e}+\bar{r}_{r}
$$

It is known [24], while the absolute motion of air particles $\mathrm{M}$ the radius-vector $\bar{r}_{e}, \bar{r}_{r}$ and $\bar{r}_{a}$ will over time $\mathrm{t}$ change in magnitude and direction according to different laws, each of these radius-vectors are variable vectors (vector-functions) that depend on the argument t: 


$$
\bar{r}_{a}(t)=\bar{r}_{e}(t)+\bar{r}_{r}(t)
$$

Equality (5) as known [24], determines the law of motion of air particles $M$ in vector form, as it allows at any time $t$ and construct the corresponding vector and find the position of moving particles of air $\mathrm{M}$.

Differentiating both parts of the vector equation (5) at time $t$, mathematical expression of the theorem of addition of velocities in complex motion in vector form [24] is obtained:

$$
\bar{v}_{a}=\bar{v}_{e}+\bar{v}_{r}
$$

where $\bar{v}_{a}$-absolute velocity of air particles (wind speed relative to the ground $\bar{v}_{w}$ ) (Fig. 2); $\bar{v}_{e}$ - the transportation velocity (the speed of the wagon $\bar{v}_{c a r}=\bar{v}$ ); $\bar{v}_{r}$ - the relative speed of air particles (see pp. $59-60$ in [24]). Otherwise, the absolute velocity is the vector sum of the transportation and relative velocities.

Here, according to [24], the direction of the velocity of the wagon $\bar{v}_{c a r}=\bar{v}$, as transportation speed $\bar{v}_{e}$, and wind $\bar{v}_{w}$, as absolute speed $\bar{v}_{a}$, and the relative speed of air particles $\bar{v}_{r}$, should have been provided, as shown in Fig. 3.

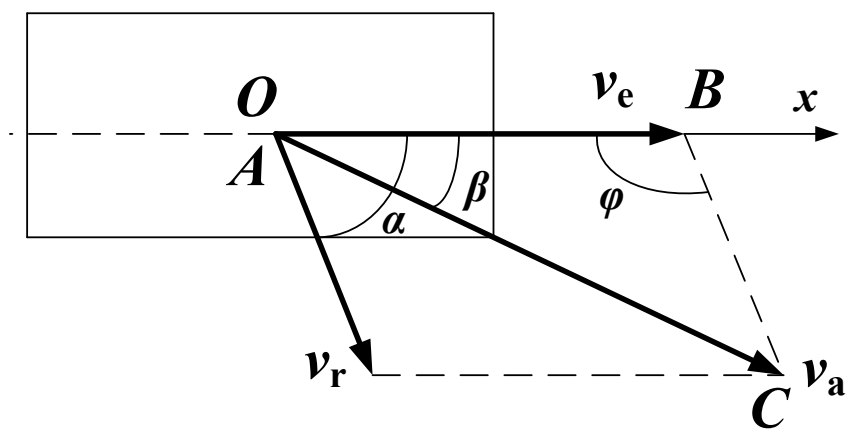

Fig. 3. The direction of the velocity vectors.

In Fig. 3 are indicated: $\bar{v}_{e}=\bar{v}-$ the average velocity of the rolling of the single wagon in the area carrying the forward speed of wagon, $\mathrm{m} / \mathrm{s} ; \bar{v}_{a}=\bar{v}_{w}$ - the wind speed relative to the ground (absolute speed of the particles is taken constant), $\mathrm{m} / \mathrm{s} ; \alpha$ is the acute angle between the relative velocity of air particles $\bar{v}_{r}$ and the direction of sliding of the wagon (axis Ox) when exposed to tailwind (see Fig. 3), rad.; $\alpha$ is the obtuse angle between the direction of the relative velocity of air particles relative to the longitudinal axis of the car (axis Ox) and transportation wagon speed $\bar{v}_{e}$ when exposed to the oncoming wind (see Fig. 3,b), rad.; $\beta$ - the angle between the wind direction and the axis of the section of the route (axis Ox), in which moves a single car at a speed of $\bar{v}_{e}$ when exposed to passing (see Fig. 3); $\bar{v}_{r}=\bar{v}_{\text {от }}-$ the relative speed of air particles (see pp. $59-60$ in [24]); $\varphi=\pi-\alpha-$ the obtuse angle complementary to the direction of the relative speed of air particles $\bar{v}_{r}$ in 
relation to the transferring velocity of the car $\bar{v}_{e}$ when exposed to tailwind (see Fig. 3) to $\pi$ rad. We make a reservation that signs of the angles $\alpha$ (the value we seek) and $\beta$ (value set). Thus, according to Fig. 3, the absolute velocity is the diagonal of a parallelogram constructed on a transportation and relative speeds.

The main error of works [19] is that the relative speed of air particles $\bar{v}_{r}$ accepted as the absolute speed of the particles (i.e. the wind speed relative to the earth as the stationary frame of reference) $\bar{v}_{a}=\bar{v}_{w}$ (see Fig. 1).

If discuss the eleventh counterexample in [17], where it was mentioned that regardless of physical sense based on the spherical law of cosines trigonometry incorrectly written formula (instead of the formula (2)), which found a relative (resultant) velocity of the wagon $\bar{v}_{r}$ with respect to the direction of wind (see formula (13) in the eleventh counterexample [17]):

$$
v_{r}^{2}=v^{2}+v_{w}^{2} \pm 2 v v_{w} \cos \beta
$$

where $\mathrm{v}=\mathrm{v}_{\mathrm{e}}-$ the average velocity of the rolling of the single wagons in the area carrying the forward speed of the wagon (the normative amount, e.g., for slides of high power in relation to the estimated phase slides from the beginning of the first braking position to the beginning of the second braking position: $\mathrm{v}=\mathrm{v}_{\mathrm{e}}=6 \mathrm{~m} / \mathrm{s}$ ), $\mathrm{m} / \mathrm{s}$;

$\mathrm{v}_{\mathrm{w}}=\mathrm{v}_{\mathrm{a}}-$ wind speed (absolute speed is taken as constant), $\mathrm{m} / \mathrm{s}$;

$\beta$ - the angle between the wind direction and the axis of the section of the route (axis $\mathrm{Ox}$ ), in which moves a single wagon at a speed of $\mathrm{v}_{\mathrm{e}}$ when exposed to tailwind (see Fig. 3 ,a) and headwind (see Fig. 3,b);

$\mathrm{V}_{\mathrm{r}}$ - the relative speed of air particles (see pp. $59-60$ in [24]).

Note that in the absence of data of wind speed to draft a hump in the rail yard in the formula (4), for example, the tailwind speed $v_{a}$ relative to earth under favorable (summer) conditions at a temperature of ambient air $\mathrm{t}=+30^{\circ} \mathrm{C}$ can be taken equal to $2.9 \mathrm{~m} / \mathrm{s}$, and the speed of the oncoming wind $v_{a}$ relative to land under adverse (winter) conditions when the ambient air temperature $\mathrm{t}=-10^{\circ} \mathrm{C}: 3.8 \mathrm{~m} / \mathrm{s}$.

We rewrite equality (7), given that it $\mathrm{v}=\mathrm{v}_{\mathrm{e}}$ and $\mathrm{v}_{\mathrm{w}}=\mathrm{v}_{\mathrm{a}}$ :

$$
v_{r}^{2}=v_{e}^{2}+v_{a}^{2} \pm 2 v_{e} v_{a} \cos \beta
$$

hence

$$
v_{r 1,2}=\sqrt{v_{e}^{2}+v_{a}^{2} \pm 2 v_{e} v_{a} \cos \beta}
$$

The equation (8) under the root sign "plus" is recommended to accept with a headwind, and the «minus» sign - with a fair wind. However, the paper will consider only the effects of wind.

As can be seen, the relative speed of air particles $v_{r}$ under the influence of tailwind and/or headwind find depending on the magnitude of the load speed of the car $v_{e}$, wind speed relative to the earth (absolute particle velocity) $v_{a}$, and the angle $\beta$ (see Fig. 3,a), i.e. $\mathrm{v}_{\mathrm{r}}=\mathrm{f}\left(\mathrm{v}_{\mathrm{e}}, \mathrm{v}_{\mathrm{a}}, \beta\right)$.

Thus, in [19] the angle $\alpha$ between the relative velocity of the air particles $v_{\mathrm{r}}$ and the direction of sliding of the carriage (axis Ox) find depending on the magnitude of the load 
speed $v_{e}$ of the wagon, the relative speed of air particles $v_{r}$ and of the angle $\beta$ (see Fig. $1 \mathrm{a}$ ), i.e. $\alpha=f\left(v_{e}, v_{r}, \beta\right)$.

1. For the proof of correctness formula (12) will present the theorem of the cosines of trigonometry when the wind (see $\triangle \mathrm{ABC}$ Fig. 3):

$$
a^{2}=b^{2}+c^{2}-2 b c \cos A .
$$

Taking into account that, according to $\triangle \mathrm{ABC}$ on Fig. $3, \mathrm{a}=\mathrm{v}_{\mathrm{a}}, \mathrm{b}=\mathrm{v}_{\mathrm{e}}, \mathrm{c}=\mathrm{v}_{\mathrm{r}}$ and $\cos \mathrm{A}=$ $\cos \varphi=\cos (\pi-\alpha)=-\cos \alpha$, we rewrite the formula (9) in the form:

$$
v_{a}^{2}=v_{e}^{2}+v_{r}^{2}+2 v_{e} v_{r} \cos \alpha
$$

To find the relative velocity of air particles $\mathrm{v}_{\mathrm{r}}$ from the last equality we get the following quadratic equation:

$$
v_{r}^{2}+2 v_{e} \cos \alpha \cdot v_{r}+\left(v_{e}^{2}-v_{a}^{2}\right)=0
$$

Solving the latter equation, obtain the final analytical formula for determining the relative speed of air particles $\mathrm{vr}$ :

$$
v_{r 1,2}=-v_{e} \cos \alpha \pm \sqrt{\left(v_{e} \cos \alpha\right)^{2}-\left(v_{e}^{2}-v_{a}^{2}\right)}
$$

As can be seen, the relative particle velocity $v_{r}$ of air when exposed to a tailwind (see Fig. 3) find depending on the magnitude of the load speed $v_{e}$ of the car and the wind speed relative to the earth (absolute particle velocity) $v_{a}$ and the angle $\alpha$, i.e. $v_{r}=f\left(v_{e}, v_{a}, \alpha\right)$.

Evidence of the incorrectness of the formula (7) and/or (8) does not require further explanation.

2. For the proof of correctness and/or absurdity of the formula (13) in the eleventh counterexample [17], where the angle $\alpha$ was found, depending on the speed $v_{e}, v_{r}$ and angle $\beta$, i.e. $\alpha=\mathrm{f}\left(\mathrm{v}_{\mathrm{e}}, \mathrm{v}_{\mathrm{r}}, \beta\right)$, when exposed to a tailwind (Fig. 4), according to $\triangle \mathrm{ABC}$, use the theorem of sinus trigonometry:

$$
\frac{a}{\sin A}=\frac{b}{\sin B}=\frac{c}{\sin C} .
$$

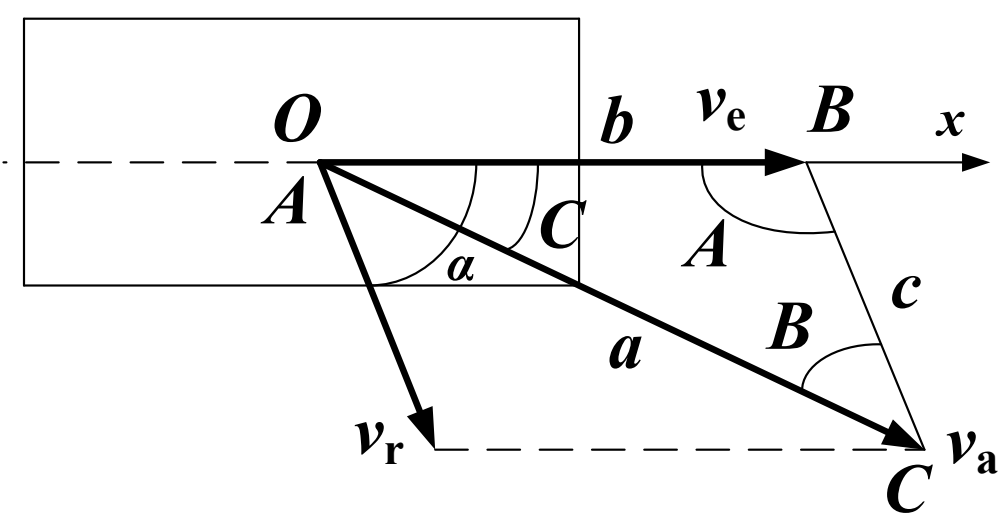

Fig. 4. On the sine theorem. 
In Fig. 4, according to $\triangle \mathrm{ABC}$ marked: $\mathrm{a}=\mathrm{v}_{\mathrm{a}}, \mathrm{b}=\mathrm{v}_{\mathrm{e}}, \mathrm{c}=\mathrm{v}_{\mathrm{r}}$ and $\sin \mathrm{A}=\sin \varphi$ or $\sin \varphi=$ $\sin (\pi-\alpha)=\sin \alpha ; \sin B=\sin [\pi-(\varphi+\beta]=\sin \{\pi-[(\pi-\alpha)+\beta]\}=\sin (\alpha-\beta) ; \sin C=\sin \beta$.

We rewrite the equality (12) in accordance with Fig. 4 signs:

$$
\frac{v_{a}}{\sin \alpha}=\frac{v_{e}}{\sin (\alpha-\beta)}=\frac{v_{r}}{\sin \beta} .
$$

Use the second and third relations in (13), we define the angle $\alpha$ depending on the $\alpha=$ $f\left(v_{e}, v_{r}, \beta\right)$ :

$$
\frac{v_{r}}{\sin \beta}=\frac{v_{e}}{\sin (\alpha-\beta)}
$$

where

$$
v_{r} \sin (\alpha-\beta)=v_{e} \sin \beta
$$

The last formula is given taking into account the formula function of the difference of angles $\alpha$ and $\beta$ :

$$
v_{r}(\sin \alpha \cos \beta-\cos \alpha \sin \beta)=v_{e} \sin \beta
$$

Dividing both parts of the last equality in $\sin \beta$, we obtain

$$
v_{r}\left(\sin \alpha \frac{\cos \beta}{\sin \beta}-\cos \alpha\right)=v_{e} .
$$

After simple mathematical calculations given the formula of a function of a single angle the last formula will be presented in the form of irrational equations to find angle $\alpha$ :

$$
\sin \alpha \cdot \operatorname{ctg} \beta-\frac{v_{e}}{v_{r}}=\sqrt{1-\sin ^{2} \alpha} .
$$

Solving an irrational equation (14), after transformation, will receive:

$$
\sin ^{2} \alpha\left(1+\cdot \operatorname{ctg}^{2} \beta\right)-2 \frac{v_{e}}{v_{r}} \sin \alpha \cdot \operatorname{ctg} \beta+\left[\left(\frac{v_{e}}{v_{r}}\right)^{2}-1\right]=0 .
$$

Further, given the formula of one function $\left(1+\operatorname{ctg}^{2} \beta\right)$ through another of the same angle $\left(\sin ^{2} \beta\right)$, we have:

$$
\sin ^{2} \alpha \frac{1}{\sin ^{2} \beta}-2 \frac{v_{e}}{v_{r}} \sin \alpha \cdot \frac{\cos \beta}{\sin \beta}+\left[\left(\frac{v_{e}}{v_{r}}\right)^{2}-1\right]=0 .
$$

Using functions of multiple angles $(\sin 2 \beta)$, after some elementary mathematical calculations, we have the following quadratic equation: 


$$
\sin ^{2} \alpha-2 \frac{v_{e}}{2 v_{r}} \sin 2 \beta \cdot \sin \alpha+\left[\left(\frac{v_{e}}{v_{r}}\right)^{2}-1\right] \sin ^{2} \beta .
$$

Solving this quadratic equation, we finally obtain the formula to determine the angle $\alpha$ :

$$
(\sin \alpha)_{1,2}=\frac{v_{e}}{2 v_{r}} \sin 2 \beta \pm \sqrt{\left(\frac{v_{e}}{2 v_{r}} \sin 2 \beta\right)^{2}-\left[\left(\frac{v_{e}}{v_{r}}\right)^{2}-1\right] \sin ^{2} \beta} .
$$

As can be seen, the obtained formula (16) of the wagon when exposed to tailwind (see Fig. 4) has a completely different but complicated form than the simple form of formula (2), which confirms the incorrectness of the formula in [19] as required.

Comparative analysis of formulas (8) and (10), (16) shows that the basic error of papers [19] and also due to the fact that the results of these studies critically analyzed [17], is that the relative speed of air particles $\bar{v}_{r}$ accepted as the absolute speed of the particles (i.e. the wind speed relative to the ground) $\bar{v}_{a}=\bar{v}_{w}$ (see Fig. 1 and 3).

For certainty, correctness and/or incorrectness performed analytical studies on the construction of mathematical models of the motion of the wagon when exposed to a tailwind we cite the following examples.

\section{Research result}

\subsection{Example of calculations 1}

For example, examine the motion of the wagon on the section of the second switching zone (Sz) the drain side slides under the influence of wind. The original data sample are: $\boldsymbol{G}=908$ - gravity wagon with load, $\mathrm{kN} ; \boldsymbol{v}_{\boldsymbol{e}}=\boldsymbol{v}_{\text {we6c } 2}=2.475$ - the speed of the wagon entrance to the investigated section of the hump, $\mathrm{m} / \mathrm{s} ; \boldsymbol{i}_{\mathbf{6} c 2}=0.002-$ the angle of slope slides Sz of hump, rad.; $\boldsymbol{l}_{\mathbf{6 c} 2}=21.0-$ length of land section Sz of hump, $\mathrm{m}$. For very good jogger will take, for example, low-sided 4-axle car, gravity $908 \mathrm{kN}$ (or 92.56 ton force); the calculated value of the basic specific resistance movement $-\boldsymbol{w}_{0}=0.5 ; \boldsymbol{v}_{\boldsymbol{a}}=2.9$ - wind speed (passing summer wind), $\mathrm{m} / \mathrm{s} ; \boldsymbol{t}=30^{\circ} \mathrm{C}$ - the estimated ambient air temperature for favorable (summer) conditions; $\boldsymbol{S}=8.5$ - the cross-sectional area of the low-sided 4-axle car, $\mathrm{m}^{2} ; \boldsymbol{c}_{\boldsymbol{x}}=1.36-$ coefficient of air resistance of the car.

\subsection{The results of the calculation in the base system Matchad [25]}

1. Substituting the original data into the formula (9) that the same formula (1), a relative speed of air particles vr1 when exposed to tailwind, $\mathrm{m} / \mathrm{s}$ :

$$
\begin{gathered}
v_{r}=\sqrt{v_{e}^{2}+v_{a}^{2}-2 v_{e} v_{a} \cos \beta}= \\
\sqrt{2.475^{2}+2.9^{2}-2 \cdot 2.475 \cdot 2.9 \cdot(0.866)}=1.451 .
\end{gathered}
$$


Make the graphical curve of the $v_{\mathrm{r} 1}=\mathrm{f}(\beta)$, by varying the angle $\beta$ between the speed of the car $\mathrm{v}_{\mathrm{e}}$ and speed of the wind relative to the ground (absolute speed of the particles) $\mathrm{v}_{\mathrm{a}}$ in the range from $10^{\circ}$ to $90^{\circ}$ in steps $\Delta \beta=5^{\circ}$ (Fig. 5).

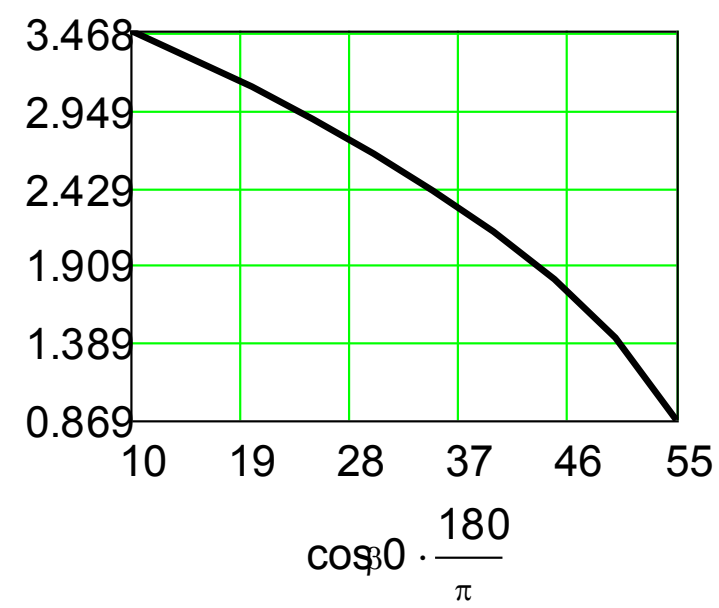

Fig. 5. The graphical curve $v_{r 1}=f(\beta)$.

Note that the graphical constructions are made in the system Mathcad [20].

As can be seen, the curve $v_{\mathrm{r} 1}=\mathrm{f}(\beta)$ the nonlinear decreasing in accordance with the formula (8). In this case $\beta \geq 59^{\circ}$ obtained integrated (i.e. cumulative) result: $\mathrm{v}_{\mathrm{r} 1}=\mathrm{I}\left(\mathrm{v}_{\mathrm{r} 1}\right)=$ $0.496 \mathrm{i}$ - the imaginary part of the numbers $\mathrm{v}_{\mathrm{r} 1}$ ( $\mathrm{i}$ - the imaginary unit), meaning that the formula (8) with given initial data is applicable only when $\beta \leq 59^{\circ}$.

2. According to the formula (2) we calculate the angle $\alpha$ between the directions of the relative velocity of air particles $v_{r}$ and load speed (or the speed of the wagon $v_{e}$ ) (axis Ox), in the following form, rad.:

$$
\sin \alpha=\frac{v_{e}}{v_{r}} \sin \beta=\frac{2.475}{1.451} \cdot 0.5=0.853,
$$

hence we have: $\alpha=\sin (\alpha) \cdot 180 / \pi=58^{\circ} 30^{\prime}$ and $\cos (\alpha)=0.5225$.

As can be seen, when exposed to a tailwind angle $\beta$ more and/or equal $\alpha$, i.e. $\beta \geq \alpha$, that is not consistent with the Fig. 1. Therefore adopted in the eleventh counterexample [17] the opposite direction of the velocity of the wagon $v_{\text {car }}$ in Fig. 1 is incorrect.

3. In the end analytical formula (16) we calculate the angle $\alpha$ between the directions of wind velocity (or absolute speed) $\mathrm{v}_{\mathrm{a}}$ and the relative speed of air particles $\mathrm{v}_{\mathrm{r}}$ if the accepted value $\beta=28^{\circ}$.

Taking initial data in the form: $v_{\mathrm{e}}=2.475$ and $\mathrm{v}_{\mathrm{r}}=1.45 \mathrm{M} / \mathrm{c}, 2 \beta=56^{\circ}$ or $\sin (2 \beta)=$ 0.848 , we obtain: 


$$
\begin{aligned}
& (\sin \alpha)_{1}=\frac{v_{e}}{2 v_{r}} \cdot \sin (2 \cdot \beta)+\sqrt{\left(\frac{v_{e}}{2 \cdot v_{r}} \cdot \sin (2 \cdot \beta)\right)^{2}-\left[\left(\frac{v_{e}}{v_{r}}\right)^{2}-1\right] \cdot \sin ^{2} \beta}= \\
= & \frac{2.475}{2 \cdot 1.45} \cdot 0.848+\sqrt{\left(\frac{2.475}{2 \cdot 1.45} \cdot 0.848\right)^{2}-\left[\left(\frac{2.475}{1.45}\right)^{2}-1\right] \cdot 0.853}=0.478,
\end{aligned}
$$

hence, we find: $\alpha=\sin (\alpha) \cdot 180 / \pi=28^{\circ} 30^{\prime}$ and $\cos (\alpha)=0.8788$.

Relative error of angle calculations $\alpha$ by formula (2) and by exact formula (17) is $\delta \alpha=$ $51.37 \%$, this confirms the incorrectness of the formula (14) in the eleventh counterexample [17].

As can be seen, taking into account the impact of tailwind direction $\beta$ wind speed relative to the earth (absolute particle speed) $v_{a}$ slightly different from the direction $\alpha$ of the relative velocity of the air particles $\mathrm{v}_{\mathrm{r}}$, for example, the angle $(\alpha-\beta)=28^{\circ} 30^{\prime}-28^{\circ}=30^{\prime}$, i.e. $\alpha>\beta$, this confirms the correctness of the derivation of formula (17) for the given initial data of the problem.

4. Let's build a graphical curve $\alpha=\mathrm{f}(\beta)$, changing angle value $\beta$ between the speed of the wagon $\mathrm{v}_{\mathrm{e}}$ and wind speed relative to the earth (absolute particle speed) $\mathrm{v}_{\mathrm{a}}$, within $10^{\circ}$ to $90^{\circ}$ in increments $\Delta \beta=5^{\circ}$ (fig. 6).

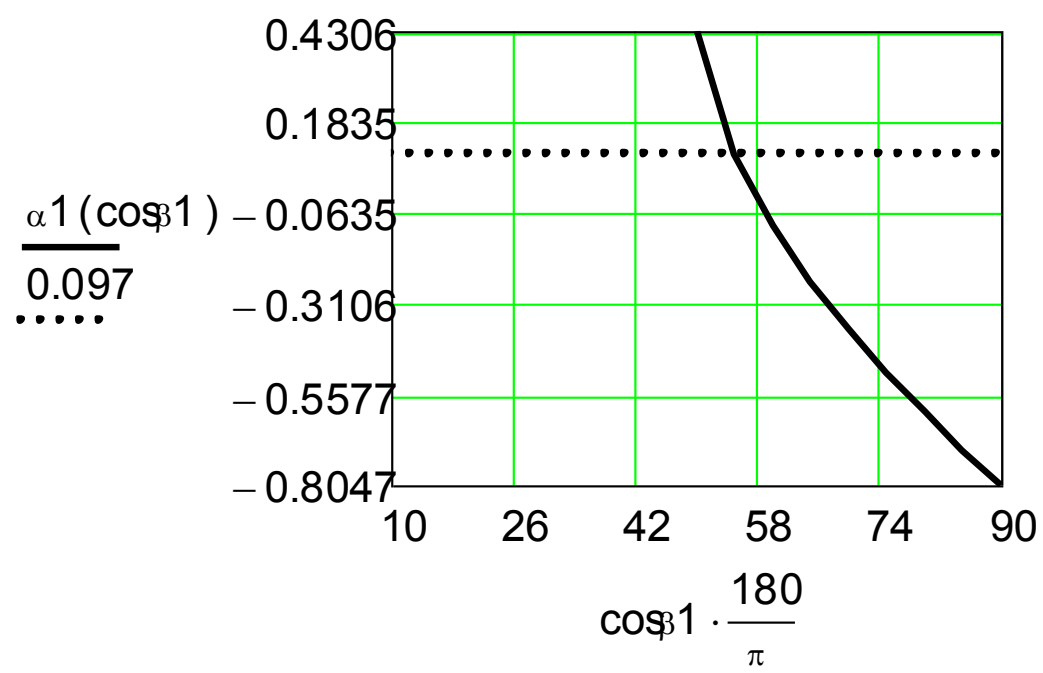

Fig. 6. The graphical curve $\alpha=f(\beta)$.

Analyzing dependencies $\alpha=\mathrm{f}(\beta)$ note decreasing their nonlinear character, starting with the value of the angle $\beta=57^{\circ} 295^{\prime}$, at which angle $\alpha=0$.

In this case $\beta \geq 49^{\circ} 16^{\prime}$ a complex result is obtained:

$$
\mathrm{v}_{\mathrm{r} 1}=\mathrm{R}\left(\mathrm{v}_{\mathrm{r} 1}\right)+\mathrm{I}\left(\mathrm{v}_{\mathrm{r} 1}\right)=0.726-0.496 \mathrm{i} \cdot 10^{-3}
$$

where $\mathrm{R}\left(\mathrm{v}_{\mathrm{r} 1}\right)=0.726^{\circ}$ - real parts of numbers $\mathrm{V}_{\mathrm{r} 1}$;

$\mathrm{I}\left(\mathrm{v}_{\mathrm{r} 1}\right)=-0.496 \mathrm{i} \cdot 10^{-3}-$ imaginary parts of numbers $\mathrm{v}_{\mathrm{r} 1}(\mathrm{i}-$ imaginary unit).

With $\beta=57^{\circ} 295^{\prime}$ : angle $\alpha=0$, and with $\beta>57^{\circ} 296^{\prime}$ angle $\alpha$ has a negative sign, which means that the formula (16) for the given initial data is applicable for $49^{\circ} 16^{\prime} \leq \beta \leq 57^{\circ} 295^{\prime}$. 
5. Determine the relative velocity of the air particles $\mathrm{v}_{\mathrm{r}}$ by the finite analytical formula (11), m/s:

$$
\begin{gathered}
v_{r 1}=-v_{e} \cos \alpha+\sqrt{\left(v_{e} \cos \alpha\right)^{2}-\left(v_{e}^{2}-v_{a}^{2}\right)}= \\
=2.475 \cdot(0.8788)+\sqrt{(2.475 \cdot(0.8788))^{2}-\left(2.475^{2}-2.9^{2}\right)}=0.474
\end{gathered}
$$

Let us calculate the second root of the equation (11):

$$
\begin{gathered}
v_{r 2}=-v_{e} \cos \alpha-\sqrt{\left(v_{e} \cos \alpha\right)^{2}-\left(v_{e}^{2}-v_{a}^{2}\right)}= \\
=2.475 \cdot(0.879)-\sqrt{(2.475 \cdot(0.879))^{2}-\left(2.475^{2}-2.9^{2}\right)}=-4.82 .
\end{gathered}
$$

As can be seen, the second root of equation (11) does not satisfy the solution of the problem, since the value of the velocity $\mathrm{v}_{\mathrm{r} 2}$ has a negative sign, i.e. $\mathrm{v}_{\mathrm{r} 2} \approx-4.8 \mathrm{~m} / \mathrm{s}$, meaning no physical meaning of calculation:

Note that the relative error of the calculation of the first root of equation (11), compared with the data of formula (8), was $\delta \mathrm{v}_{\mathrm{r} 1} \approx 67.4 \%$, what is unacceptable for engineering calculations $(\approx 5 \%)$. However, it should be borne in mind that the formula (8) is incorrect.

Hence it is clear that in the $[13-15,19]$ performed on the basis in the eleventh counterexample [17] contains fallible materials that do not correspond to the principles of theoretical mechanics [24].

\section{Scope of results}

Results of study can be used at processing of the standard and technical document on design of sorting devices on the railroads and corrections in the description of dynamics of rolling down of the wagon from marshalling hump.

\section{General conclusions}

1. Comparative analysis of formulas (2), (7) and (10), (16) shows that the gross error of works [17], is that the relative velocity of air particles $\bar{v}_{r}$ taken as the absolute velocity of the particles (i.e., the wind speed relative to the earth) $\bar{v}_{a}=\bar{v}_{w}$ (see Fig. 1 and 3).

2. Gross errors made in the construction of a mathematical model of accounting for the impact of tailwind and / or headwind in [19], reduces the practical significance of the use in the hump design and technological calculations of the formula (3) to find the resistivity of the movement of the wagon from the air and wind $\boldsymbol{\omega}_{a e}$ (and $\boldsymbol{w}_{a e}$, obtained on the basis of empirical dependences taking into account aerodynamic parameters (streamlining) of wagons (see the middle column on page 37 in [17]).

3 . In the $[13-15,19]$ made on the basis of [17] contains fallible materials that do not correspond to the principles of theoretical mechanics [24]. 
1. C. Zhang, Y. Wei, G. Xiao, Z. Wang, J. Fu, Proc. of Second Intern. Conf. on Transportation and Traffic Studies, 285-290 (2000) doi: 10.1061/40503(277)45

2. S. Zářecký, J. Grúň, J. Žilka, Transport Problems 3(4), 87-95 (2008) http://transportproblems.polsl.pl/pl/Archiwum/2008/zeszyt4/2008t3z4_13.pdf

3. T. Judge, Railway Age 5, 33-34 (2007)

4. C. Zhang, Y. Li, Proc. of Intern Conf. «Optoelectronics and Image Processing (ICOIP)» 2, 262-265 (2010) doi: 10.1109/ICOIP.2010.274

5. O. Kondo, Y. Yamazaki, Nippon Steel \& Sumitomo Metal Technical Report 105, 7783 (2013)

6. C.T. Dick, J.R. Dirnberger, Joint Rail Conference American Society of Mechanical Engineers, 04022014 (2014)

7. K. Turanov, Global Journal of Researches in Engineering: A. Mechanical and Mechanics Engineering 14(1), 1-9 (2014)

8. S.O. Bantukova, Eastern-European Journal of Enterprise Technologies 3.3(75), 4-9 (2015) doi: 10.15587/1729-4061.2015.42400

9. M.G. Bardossy, Proceedings of the 5th International Conference on Simulation and Modeling Methodologies, Technologies and Applications, SCITEPRESS - Science and and Technology Publications, 493-500 (2015)

10. N. Boysen, S. Emde, M. Fliedner, OR Spectrum 38(1), 207-233 (2016) doi: 10.1007/s00291-015-0412-0

11. C. Lu, J. Shi, Advances in Structural Engineering 34(1), 136943321987057 (2019) doi: $10.1177 / 1369433219870573$

12. O. Polach, Wear 258(7-8), 992-1000 (2005) doi: 10.1016/j.wear.2004.03.046

13. V. Bobrovskyi, D. Kozachenko, A. Dorosh, E. Demchenko, T. Bolvanovska, A. Kolesnik, Transport Problems, IV Symposium of Young Researchers, 632-640 (2015)

14. V. Bobrovskyi, D. Kozachenko, A. Dorosh, E. Demchenko, T. Bolvanovska, A. Kolesnik, Transport Problems 11(1), 147-155 (2016) doi: 10.20858/tp.2016.11.1.14

15. D. Kozachenko, V. Bobrovskyi, Y. Demchenko, Journal of Modern Transportation 26(3), 189-199 (2018) https://doi.org/10.1007/s40534-018-0161-2

16. K. Turanov, A. Gordiienko, Open Access Library Journal 2, e1912 (2015) doi: http://dx.doi.org/10.4236/oalib.1101912

17. Kh. Turanov, Transport information Bulletin 3(237), 29 - 36 (2015) ISSN 2072-8115

18. V.M. Rudanovskij, Transport information Bulletin 6(252), 19-28 (2016) ISSN 20728115.

19. Y.O. Pozojskij, V.M. Rudanovskij, V.A. Kobzev, I.P. Starshov, Transport information Bulletin 2(272), 35-38 (2018) ISSN 2072-8115

20. Kh.T. Turanov, A.A. Gordienko, MATEC Web of Conferences conference proceedings, 02027 (2018)

21. K. Turanov, E.Timukhina, A. Gordienko, TransSiberia 2019 AISC 1115, 703-716 (2020) https://doi.org/10.1007/978-3-030-37916-2_69

22. J. Prokop, Sh. Myojin, Memoirs of the Faculty of Engineering Okayama University 27(2), 41-58 http://ousar.lib.okayama_u.ac.jp/file/15404/Mem_Fac_Eng_OU_27_2_41.pdf

23. J. Prokop, Sh. Myojin, Memoirs of the Faculty of Engineering, Okayama University 27(2), 59-71 (1993) http://ousar.lib.okayamau.ac.jp/file/15406/Mem_Fac_Eng_OU_27_2_59.pdf 
24. K.L. Komarov, Theoretical mechanics in railway transport problems (Nauka, Novosibirsk, 2004)

25. D.V. Kiryanov, Tutorial MathCAD 13 (Samouchitel MathCAD 13) (BHV-Peterburg, Saint-Petersburg, 2006) 